\title{
Are steroids required in the treatment of ganglion impar blockade in chronic coccydynia? a prospective double-blinded clinical trial
}

\author{
Savas Sencan ${ }^{1}$, Ipek Saadet Edipoglu ${ }^{1}$, Fatma Gul Ulku Demir ${ }^{2}$, Gunay Yolcu ${ }^{3}$, and Osman Hakan Gunduz ${ }^{1}$ \\ 'Division of Pain Medicine, Department of Physical Medicine and Rehabilitation, Faculty of Medicine, Marmara University, Istanbul, Turkey \\ ${ }^{2}$ Department of Physical Medicine and Rehabilitation, Kayseri City Hospital, Kayseri, Turkey \\ ${ }^{3}$ Department of Physical Medicine and Rehabilitation, Faculty of Medicine, Marmara University, Istanbul, Turkey
}

Received June 27, 2019

Revised August 2, 2019

Accepted August 9, 2019

\section{Correspondence}

Ipek Saadet Edipoglu

Marmara Üniversitesi Tıp Fakültesi

Pendik Eğitim ve Araştırma Hastanesi

Ağıı Bilim Dalı Fevzi Çakmak Mahallesi,

Muhsin Yazıcıoğlu Cd No:10, 34899

Pendik/Istanbul, Turkey

Tel: +90-216-625-45-45

Fax: +90-216-414-47-31

E-mail: dripeks@yahoo.com

\section{Key Words}

Adrenal Cortex Hormones

Anesthetics, Local

Beck Test

Coccygeal Pain

Depression

Ganglion Impar Blockade

Pain

Rating Scale
Background: Ganglion impar blockade is a reliable and effective treatment option used in patients with coccydynia. Our primary objective was to specify the role of corticosteroids in impar blockade. We compared applications of local anesthetic with the local anesthetic + corticosteroid combination in terms of treatment efficiency in patients with chronic coccydynia.

Methods: Our study was a prospective randomize double-blind study. The patients were divided into 2 groups after randomization. The first group (group SL) was made up of patients where a corticosteroid + local anesthetic were used during ganglion impar blockade. In the second group (group L) we used only local anesthetic. We evaluated numeric rating scale (NRS) and Beck depression scale, which were employed before the procedure and in 1st and 3rd months after the procedure.

Results: Seventy-three patients were included in the final analysis. We detected a significantly greater decrease in NRS values in the 1st month in group SL than in group $L(P=0.001)$. In the same way, NRS values in the 3rd month were significantly lower in the group with steroids $(P=0.0001)$. During the evaluation of the Beck test, we detected significantly greater decreases in the 1st month $(P=0.017)$ and 3rd month $(P=0.021)$ in the $S L$ group than in the $L$ group.

Conclusions: Ganglion impar blockade decreases pain in the treatment of chronic coccydynia and improve depression. Addition of steroids in a ganglion impar blockade is required for treatment response that should accumulate over a long period of time.

\section{INTRODUCTION}

Coccydynia, also known as coccygodynia, is defined as localized pain in the region of coccyx [1]. Although its incidence isn't completely known, the risk of coccydynia progress is higher in the female sex and when obesity is present [1]. Despite the fact that many explanations in its etiology are related to trauma, there are also idiopathic cases [2]. In $90 \%$ of patients, recovery from coccydynia has a chance of being rendered either without treatment or through con- servative treatments. In some patients, symptoms might last for a long time and result in psycho-social disorders and a dramatic decrease in the quality of life $[3,4]$. In spite of the fact that interventional treatment works in such patients, there is no standard treatment algorithm in coccydynia, and the few existing studies contain conflicting results $[1,5]$.

Ganglion impar blockade, one of the interventional treatment methods, is a reliable treatment option conducted frequently in patients with coccydynia and renders a (a) This is an open-access article distributed under the terms of the Creative Commons Attribution Non-Commercial License (http://creativecommons.org/licenses/by-nc/4.0/), which permits unrestricted non-commercial use, distribution, and reproduction in any medium, provided the original work is properly cited.

(C) The Korean Pain Society, 2019
Author contributions: Savas Sencan: Study conception; Ipek Saadet Edipoglu: Writing/manuscript preparation; Fatma Gul Ulku Demir: Data curation; Gunay Yolcu: Data curation; Osman Hakan Gunduz: Supervision. 
significant amount of decrease in pain. Although ganglion impar blockade, first defined by Plancarte et al. [6], has evolved over time, a fluoroscopy guided transsacrococcygeal approach is often preferred in our day. Local anesthetics or a corticosteroid in addition to local anesthetics are used for the purpose of the blockade. The blockade effect through local anaesthesia is most generally applied for diagnostic purposes, for purposes of radiofrequency ablation, or for prognostic purposes in patients where neurolysis via phenol/alcohol is planned. The use of local anesthetics by themselves alone for therapeutic purposes is seldom preferred and corticosteroids are generally used in addition to local anesthetics. Due to their analgesic, anti-inflammatory and neuromodulatory effects, corticosteroids are commonly used for therapeutic purposes $[7,8]$.

There are studies in the literature regarding treatment results of impar blockade conducted via the mixture of local anesthetics and corticosteroids in patients with chronic coccydynia [9-11]. Only a small number of case presentations and case series have been reported regarding blockade through a local anesthetic by itself $[12,13]$. In spite of this, as far as we know, there is no study expounding on the effect of local anesthetics alone or the mixture of local anesthetics and corticosteroids on treatment results. Besides, the fact that there are no studies encompassing comparison in terms of the treatment results of ganglion impar blockade in previous studies makes it hard to make an objective evaluation about the efficacy of this kind of treatment.

In the light of such findings, our primary objective in this study was to specify the role of corticosteroids in impar blockade through a comparison of applications of local anesthetic with a mixture of a local anesthetic and corticosteroids in terms of their responses towards treatment in patients with chronic coccydynia. Our secondary objective was to research the effect of impar blockade on pain and psychological frame of mind in chronic coccydynia.

\section{MATERIALS AND METHODS}

Our study was a prospective randomized double-blind study carried out in a duration of 1 year between 2017 to 2018. Ethical committee approval was obtained from the Committee of Ethics of Erciyes University on March 17, 2017 through a judgement numbered 2017/62. Patients in which a ganglion impar blockade was to be conducted after they were diagnosed with chronic coccydynia in the pain clinic in the subsequent stage, with clinical and physical examination, as well as visualization methods, were included in the study. Other inclusion criteria were being between the ages of 18 and 75, and not having ben- efited from conservative treatment methods. Verbal and written informed consent were obtained from all patients participating in the study. Patients not granting approval were excluded. In addition, patients with a history of ganglion impar blockade carried out in the last 3 months, patients with a history of repetitive ganglion impar blockade, patients with systemic and/or local infections, patients with a history of allergy to contrast material and/or local anaesthetic substances, patients with a history of malignancy, patients with bleeding diathesis, patients with a known history of any psychiatric disorder, patients with acute fractures, and patients with a history of pregnancy were excluded from the study.

The patients were divided into 2 groups after being randomized through a computer program. The first group (group SL) was made up of patients where a corticosteroid (methylprednisolone) mixed with local anesthetic (bupivacaine) were used during the ganglion impar blockade. The second group (group L) was made up of patients where only a local anesthetic (bupivacaine) was used. The patients did not know in any way which treatment group they were in, and, moreover, the doctor administering the drug did not know which drug he was given. In this way, our study was conducted with a double blind design. The drug to be administered was determined randomly, and it was prepared by the supervisor of the study, placed in an unlabelled, opaque, injection syringe in a separate room, and given to the doctor administering the treatment. In this way, both the doctor and patients were blinded to the administered drug.

\section{Assessment scales}

In addition to the processing of the demographic data of the patients participating in this study (age, sex, duration of symptoms, etc.), the numeric rating scale (NRS) was employed before the procedure and in the 1st hour, and 1st and 3rd months after the procedure; Beck depression scale was employed before the procedure and in the 1st and 3rd months after the procedure. The NRS is a frequently used method in measuring the severity of the pain, and in monitoring it. It is an 11-point scale, in which ' 0 ' means 'no pain' and ' 10 ' means 'the most severe pain possible' and the patient is asked to score his/her pain between 0 and 10 .

For the purpose of obtaining data about the psychological frame of mind and existence of depression in the patients, the Beck depression scale, which has been validated in the Turkish language, was employed [14]. There are 21 questions in the application of this test and there is a scoring system in each question that ranges from 0 to 3 . The total scoring ranges from 0 to 63 and in proportion to rising scores, it is assumed that there is an exacerbation towards 
depression in the patient's mood.

In this study, the assistant conducting all the interviews had no knowledge of which groups each patient belonged to.

\section{Intervention}

Ganglion impar blockade was conducted with the aid of a fluoroscope by a pain medicine expert with at least 10 years of experience in this field. The patients were made to lie down in a prone position and their intergluteal regions were made aseptic. After the sacrococcygeal joint was visualized with the fluoroscope, local anaesthesia was applied at the sacrococcygeal junction via $3 \mathrm{~mL} 2 \%$ prilocaine, and the infiltration was applied to both cutaneous and subcutaneous tissues. The sacrococcygeal joint was pierced with a 22-gauge spinal needle and the ganglion impar was reached. After a $1 \mathrm{~mL}$ injection of non-ionic contrast medium, there was dye dispersion, and an image of an "inverse comma" was detected in the lateral visualization; $3 \mathrm{~mL} 0.5 \%$ bupivacaine, $2 \mathrm{~mL}$ of saline, and $40 \mathrm{mg}$ of methylprednisolone were injected into the patients in group SL. Only $3 \mathrm{~mL} 0.5 \%$ of bupivacaine and $2 \mathrm{~mL}$ of saline were injected to the patients in group $\mathrm{L}$.

\section{Statistical analysis}

The program IBM SPSS ver. 21.0 (IBM Corp., Armonk, NY) was used for statistical analyses. During the assessment of the data, the Mann-Whitney $U$-test was used in the between-group comparison of non-parametric quantitative data, and the student $t$-test was used in the comparison of qualitative data in addition to the usage of descriptive statistical methods (mean, standard deviation, frequency, and rate). Significance was accepted to be at the level of $P<0.05$. The PS Power and Sample Size ver. 3.1.2 (Stata Corp., College Station, TX) was used for the analysis of the number of patients. At least 68 patients were required for an $\alpha$ of 0.05 and a power of 0.80 when we anticipated a $15 \%$ change in the NRS scores. Since possible dropouts were expected at high numbers, it was concluded that 87 patients in total should be included in the study.

\section{RESULTS}

One hundred twenty-eight individuals were subjected to evaluation in the previous stage of the study. Seventythree patients were included in the final analysis after applying the exclusion criteria, as well as excluding those refusing to participate, and those lost during the monitoring process (Fig. 1). Thirty-four individuals (46.6\%) were placed into group SL. We reported the median age of our patients to be $38.2 \pm 9.2$ years and 63 of them $(86.3 \%)$ were female. Their median body mass index was $26.4 \pm 4.3 \mathrm{~kg} /$ $\mathrm{m}^{2}$. In Table 1, we see that no significant difference was

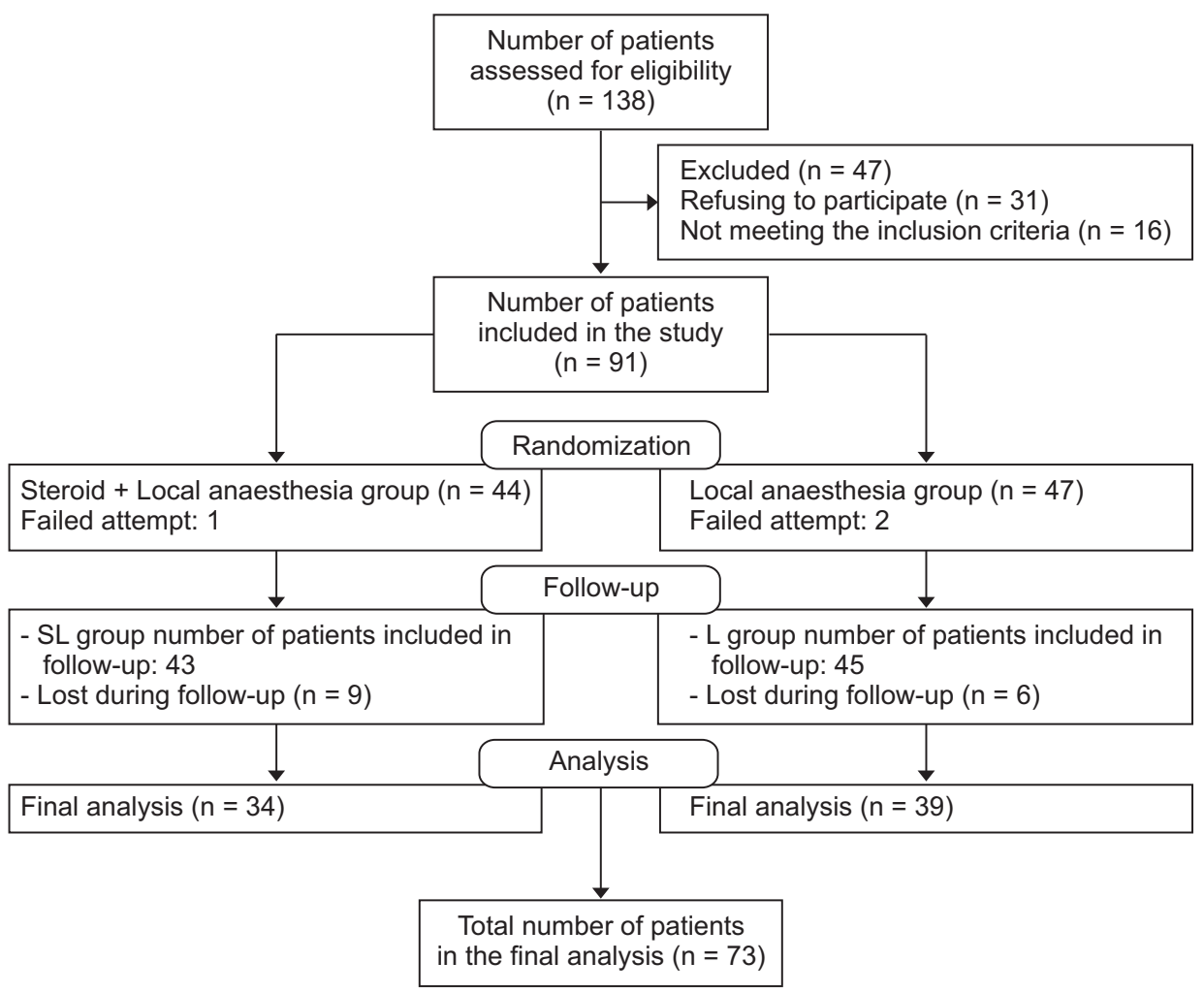

Fig. 1. Flow diagram. 
detected between the two groups in terms of demographic data and clinical findings before the procedure.

In all checks after the procedure in both groups, significant decreases in NRS scores and Beck depression scores were determined (Table 2). During the comparison of the groups, we detected no significant difference between the groups in the categories of pre-procedure NRS scores and those reported after 1 hour $(P>0.05)$. However, we detected a significantly greater decrease in NRS values at 1 month in group SL than in group $\mathrm{L}(P=0.001)$. In the same way, the NRS values at 3 months were significantly lower in group SL $(P=0.0001)$. During the evaluation of the Beck test, while there was no difference between the groups before the procedure, we detected significant decreases at 1 month $(P=0.017)$ and 3 months $(P=0.021)$ in the SL group in comparison to the L group (Table 3 ).

\section{DISCUSSION}

In this study we compared the effectiveness of ganglion

Table 1. Comparison of Groups in Terms of Demographic Data and Clinical Findings

\begin{tabular}{lccc}
\hline \multicolumn{1}{c}{ Variable } & $\begin{array}{c}\text { Group SL } \\
(\mathrm{n}=34)^{\mathrm{a}}\end{array}$ & $\begin{array}{c}\text { Group L } \\
(\mathrm{n}=39)^{\mathrm{b}}\end{array}$ & P value \\
\hline Body mass index $\left(\mathrm{kg} / \mathrm{m}^{2}\right)$ & $26.9 \pm 4.6$ & $25.9 \pm 4.0$ & 0.310 \\
Age $(\mathrm{yr})$ & $38.1 \pm 10.0$ & $38.3 \pm 8.6$ & 0.941 \\
Sex & & & 0.499 \\
$\quad$ Female & $28(82.4)$ & $35(89.7)$ & \\
$\quad$ Male & $6(17.7)$ & $4(10.3)$ & \\
Symptom duration $(\mathrm{mo})$ & $16.0 \pm 12.7$ & $19.5 \pm 15.7$ & 0.307 \\
History of trauma & & & 0.241 \\
$\quad$ Yes & $21(61.8)$ & $17(43.6)$ & \\
$\quad$ No & $13(38.2)$ & $22(56.4)$ & \\
Beck test before the procedure & $19.9 \pm 9.0$ & $22.8 \pm 12.2$ & 0.125 \\
NRS before the procedure & $7.8 \pm 1.4$ & $8.1 \pm 1.4$ & 0.487 \\
\hline
\end{tabular}

Values are presented as mean \pm standard deviation or number (\%). NRS: numeric rating scale.

${ }^{a}$ Steroid + Local anaesthesia group, ${ }^{b}$ only local anaesthesia group. impar blockade with local anesthetics alone to that with steroids on pain and mood in patients with chronic coccydynia. As a result, ganglion impar blockade either with or without steroids was discovered to improve depression scores and to decrease pain in patients with chronic coccydynia. Nonetheless, while the decrease in pain scores was similar in both groups in checks at 1 hour, it was discovered that in group SL, a decrease in pain scores and improvement in depression scores were significantly higher in checks at 1 and 3 months.

The analgesic efficacy of corticosteroids has been known for a long time, and they play a role in adjuvant therapy. In spite of the fact that the strongest mechanism responsible for analgesia from corticosteroids is thought to be their anti-inflammatory effects, it is not known why the use of corticosteroid produces a better analgesic effect [7]. It has also been shown in experimental studies that corticosteroids have direct neural antinociceptive effects in their analgesic impact [15]. The anti-inflammatory effects are the result of the pleiotropic effects of glucocorticoid receptors on multiple signal pathways of corticosteroids [15]. These lipophilic hormones pass cytoplasmic membranes and regulate gene expression. They upregulate the transcription of anti-inflammatory genes by being translocated on the nucleus in the subsequent stage of the steroid receptor complex $[15,16]$. Glucocorticoids also inhibit the expression in many pro-inflammatory factors contain-

Table 3. Comparison of Groups in Terms of NRS and Beck Test

\begin{tabular}{lccl}
\hline \multicolumn{1}{c}{ Variable } & Group SL $(\mathrm{n}=34)^{\mathrm{a}}$ & Group L $(\mathrm{n}=39)^{\mathrm{b}}$ & $P$ value \\
\hline NRS 1 hr & $2.5 \pm 2.9$ & $1.6 \pm 1.5$ & 0.112 \\
NRS 1 mo & $2.4 \pm 2.5$ & $4.7 \pm 3.0$ & $0.001^{*}$ \\
NRS 3 mo & $2.9 \pm 2.7$ & $6.2 \pm 2.9$ & $0.0001^{*}$ \\
Beck test 1 mo & $11.5 \pm 8.1$ & $16.9 \pm 10.6$ & $0.017^{*}$ \\
Beck test 3 mo & $12.1 \pm 8.6$ & $17.8 \pm 11.2$ & $0.021^{*}$ \\
\hline
\end{tabular}

Values are presented as mean \pm standard deviation.

NRS: numeric rating scale.

${ }^{*} P<0.05$ was accepted as significant.

a Steroid + Local anaesthesia group, 'only local anaesthesia group.

Table 2. Comparison of NRS and Beck Depression Scores between In-Groups during Follow-Ups before the Procedure and after the Treatment

\begin{tabular}{|c|c|c|c|c|}
\hline Variable & Group SL $(n=34)^{a}$ & $P$ value* & Group L $(n=39)^{b}$ & $P$ value* \\
\hline NRS before the procedure vs. $1 \mathrm{hr}$ & $5.5 \pm 2.9$ & 0.0001 & $6.5 \pm 1.6$ & 0.0001 \\
\hline NRS before the procedure vs. 1 mo & $5.4 \pm 2.7$ & 0.0001 & $3.4 \pm 2.7$ & 0.0001 \\
\hline NRS before the procedure vs. 3 mo & $4.9 \pm 2.9$ & 0.0001 & $1.9 \pm 2.6$ & 0.0001 \\
\hline Beck test pre-procedure vs. 1 mo & $5.9 \pm 12.5$ & 0.010 & $7.0 \pm 10.0$ & 0.001 \\
\hline Beck test pre-procedure vs. 3 mo & $5.0 \pm 14.0$ & 0.045 & $6.4 \pm 10.9$ & 0.002 \\
\hline
\end{tabular}

Values are presented as mean \pm standard deviation.

NRS: numeric rating scale.

$* P<0.05$ was accepted as significant.

a Steroid + Local anaesthesia group, ${ }^{\text {b }}$ only local anaesthesia group. 
ing enzymes related with pro-inflammatory cytokines, chemokines, and inflammation $[15,17,18]$. Such processes transpire slowly, and they are held responsible for the delayed effect lasting for days and weeks after steroid injections, unlike local anesthetics, commonly used in conjunction with steroids, whose effects possibly commence instantly. In our study, the fact that the decrease in pain scores was higher in group SL at 1 and 3 months might be related to these effects of late commencement, although in both groups the decrease in pain scores was similar 1 hour after the injection.

Because glucocorticoid receptors are richly expressed in the hippocampus and amygdala, glucocorticoids also manifest behavioural and antidepressant effects [19]. This situation clarifies why there was more improvement in the depression scores at 1 and 3 months in group SL compared to group L. Besides, considering the decrease in pain scores in group SL was higher at 1 and 3 months, the fact that the improvement in depression scores was higher in conjunction with decreased pain are findings that are mutually supportive.

These wide-ranging effects of steroids, and the positive results propelled by them, have resulted in frequent studies on the administration of additional steroids in conjunction with local anesthetics in ganglion impar blockade on patients with chronic coccydynia in the literature. In one of these studies carried out by Gunduz et al. [9], a retrospective pilot study encompassing 22 chronic coccydynia patients on whom ganglion impar blockade was conducted via the transsacrococcygeal pathway, it was reported that there was a success rate of $82 \%$ after the first injection and relief from pain lasting, on average, for 6 months. In another recent study, Gonnade et al. [20] prospectively examined 31 patients with chronic coccydynia on whom ganglion impar blockade had been carried out, and they evaluated their pain through the NRS and their functionality through the oswestry disability index (ODI) at $2,4,12$, and 24 weeks [21]. The authors have reported a profound decrease in pain levels, a decrease in NRS scores, and noticeable positive results in the ODI [20]. Our study, in nature, supports the results of both studies, where additional steroids were administered in conjunction with local anesthetics for the purpose of ganglion impar blockade. On the other hand, our study, also encompassing a patient group on whom local anaesthesia alone was used, is important in that it shows the role of steroid addition in treatment responses.

In addition to the blockade effect of local anesthetics on characteristic voltage-dependent $\mathrm{Na}^{+}$channels, they have modulatory effects on many other neuronal channels [21]. Bupivacaine, a reliable local anesthetic with a prolonged effect, has been shown to cause the inhibition of the flow of N-methyl-D-aspartate (NMDA) receptors, which plays an important role in central sensitization dependent on chronic pain [21]. Therefore, the fact that the decrease in pain scores in group L in our study, in whom pain was more noticeable at 1 hour, continued over the course of 3 months with an improvement in depression scores might be related to the block the central sensitization by inhibition of NMDA receptor by bupivacaine in conjunction with the chronic pain process in our patients. These results reveal that ganglion impar blockade through a local anesthetic alone might be an alternative in patient groups such as those with uncontrolled blood sugar regulation, where immune suppression from the use of steroids is risky.

As shown in the 20-patient case series presentations by Buttaci et al. [12], in the subsequent stage of ganglion impar blockade with only a local anesthetic, an average decrease in pain at the rate of $20 \%$ to $75 \%$, generally lasting weeks or months was recorded after each injection. It has been reported that the analgesic effect decreased over time in many of the patients, and the pain response to repetitive injections was better [12]. Le Clerc et al. [22] conducted 220 ganglion impar blockades on 83 patients in a retrospective study they carried out with patients with chronic pelvic and perineal pain. They felt the need to carry out a second blockade on 75 patients and a third blockade on 62 patients. They conducted these blockades using only a local anesthetic, and they did more than one blockade in many of the patients to exert an effective, prolonged response [22]. We are of the opinion that if they had used additional steroids as we did in our study, the need for repetitive injections might have been removed as the result of obtaining a higher rate of pain decrease in a single blockade in a similar way to our results.

Our study has some limitations due to the short followup time, the fact that functional assessment couldn't be made because of the absence of specific scales directed at chronic coccydynia, and that our study did not include a real placebo control group because of ethical reasons. Nonetheless, as far as we can tell, because it is the first comparative, prospective double-blinded study regarding ganglion impar blockade, and considering its results, it is a valuable study.

Ganglion impar blockade performed either with or without steroids renders a decrease in pain in the treatment of chronic coccydynia and an improvement in depressive mood. Nonetheless, the addition of steroids to the ganglion impar blockade is required for a treatment response that should accumulate over a long period of time. For this reason, steroids should be added as an adjuvant agents in each ganglion impar blockade performed for therapeutic reasons in patients where the use of steroids isn't contraindicated. 


\section{CONFLICT OF INTEREST}

No potential conflict of interest relevant to this article was reported.

\section{ORCID}

Savas Sencan, https://orcid.org/0000-0001-8150-0581

Ipek Saadet Edipoglu, https://orcid.org/0000-0002-3510-5991

Fatma Gul Ulku Demir, https://orcid.org/0000-0003-4160-8568

Gunay Yolcu, https://orcid.org/0000-0002-8545-7821

Osman Hakan Gunduz, https://orcid.org/0000-0002-3214-803X

\section{REFERENCES}

1. Nathan ST, Fisher BE, Roberts CS. Coccydynia: a review of pathoanatomy, aetiology, treatment and outcome. J Bone Joint Surg Br 2010; 92: 1622-7.

2. Maigne JY, Guedj S, Straus C. Idiopathic coccygodynia. Lateral roentgenograms in the sitting position and coccygeal discography. Spine (Phila Pa 1976) 1994; 19: 930-4.

3. De Andrés J, Chaves S. Coccygodynia: a proposal for an algorithm for treatment. J Pain 2003; 4: 257-66.

4. Maroy B. Spontaneous and evoked coccygeal pain in depression. Dis Colon Rectum 1988; 31: 210-5.

5. Maigne JY, Chatellier G, Faou ML, Archambeau M. The treatment of chronic coccydynia with intrarectal manipulation: a randomized controlled study. Spine (Phila Pa 1976) 2006; 31: E621-7.

6. Plancarte R, Amescua C, Patt RB, Allende S. Presacral blockade of the ganglion of Walther (ganglion impar). Anesthesiology 1990; 73: A751.

7. Leppert W, Buss T. The role of corticosteroids in the treatment of pain in cancer patients. Curr Pain Headache Rep 2012; 16: 307-13.

8. Carr CM, Plastaras CT, Pingree MJ, Smuck M, Maus TP, Geske JR, et al. Immediate adverse events in interventional pain procedures: a multi-institutional study. Pain Med 2016; 17: $2155-61$.

9. Gunduz OH, Sencan S, Kenis-Coskun O. Pain relief due to transsacrococcygeal ganglion impar block in chronic coccy- godynia: a pilot study. Pain Med 2015; 16: 1278-81.

10. Wray CC, Easom S, Hoskinson J. Coccydynia. Aetiology and treatment. J Bone Joint Surg Br 1991; 73: 335-8.

11. Sencan S, Cuce I, Karabiyik O, Demir FU, Ercalik T, Gunduz $\mathrm{OH}$. The influence of coccygeal dynamic patterns on ganglion impar block treatment results in chronic coccygodynia. Interv Neuroradiol 2018; 24: 580-5.

12. Buttaci C, Foye PM, Stitik TP. Coccygodynia successfully treated with ganglion Impar blocks: a case series. Am J Phys Med Rehabil 2005; 85: 783-4.

13. Turchan A, Fahmi A, Subianto H. Impar ganglion block with combination of neurolysis drugs and radiofrequency thermocoagulation for perineal pain. Asian J Neurosurg 2018; 13: 838-41.

14. AktürkZ, Dağdeviren N, Türe M, Tuğcu C. The reliability and validity analysis of the Turkish version of Beck Depression Inventory for primary care. Turk Aile Hek Derg 2005; 9: 11722.

15. Schilling LS, Markman JD. Corticosteroids for pain of spinal origin: epidural and intraarticular administration. Rheum Dis Clin North Am 2016; 42: 137-55.

16. Rhen T, Cidlowski JA. Antiinflammatory action of glucocorticoids--new mechanisms for old drugs. N Engl J Med 2005; 353: $1711-23$

17. Markman JD, Kress BT, Frazer M, Hanson R, Kogan V, Huang JH. Screening for neuropathic characteristics in failed back surgery syndromes: challenges for guiding treatment. Pain Med 2015; 16: 520-30.

18. De Bosscher K, Vanden Berghe W, Haegeman G. The interplay between the glucocorticoid receptor and nuclear factorkappaB or activator protein-1: molecular mechanisms for gene repression. Endocr Rev 2003; 24: 488-522.

19. Bair MJ, Robinson RL, Katon W, Kroenke K. Depression and pain comorbidity: a literature review. Arch Intern Med 2003; 163: 2433-45.

20. Gonnade N, Mehta N, Khera PS, Kumar D, Rajagopal R, Sharma PK. Ganglion impar block in patients with chronic coccydynia. Indian J Radiol Imaging 2017; 27: 324-8.

21. Paganelli MA, Popescu GK. Actions of bupivacaine, a widely used local anesthetic, on NMDA receptor responses. J Neurosci 2015; 35: 831-42.

22. Le Clerc QC, Riant T, Levesque A, Labat JJ, Ploteau S, Robert $\mathrm{R}$, et al. Repeated ganglion impar block in a cohort of $83 \mathrm{pa}$ tients with chronic pelvic and perineal pain. Pain Physician 2017; 20: E823-8. 